\title{
Influence of Hierarchic Structure on the Moisture Permeability of Biomimic Woven Fabric Using Fractal Derivative Method
}

\author{
Jie Fan, ${ }^{1,2,3}$ Na Zhu, ${ }^{1}$ Li-li Wang, ${ }^{2}$ Zhi Liu, ${ }^{2}$ Chang-Yuan Wang, ${ }^{2}$ and Yong Liu ${ }^{1,2}$ \\ ${ }^{1}$ Key Laboratory of Advanced Textile Composites, Ministry of Education of China, Tianjin 300387, China \\ ${ }^{2}$ School of Textiles, Tianjin Polytechnic University, Tianjin 300387, China \\ ${ }^{3}$ National Engineering Laboratory for Modern Silk, College of Textile and Engineering, Soochow University, Suzhou 251123, China
}

Correspondence should be addressed to Jie Fan; fanjie@tjpu.edu.cn and Yong Liu; liuyong@tjpu.edu.cn

Received 16 July 2014; Accepted 7 September 2014

Academic Editor: Xiao-Jun Yang

Copyright (C) 2015 Jie Fan et al. This is an open access article distributed under the Creative Commons Attribution License, which permits unrestricted use, distribution, and reproduction in any medium, provided the original work is properly cited.

\begin{abstract}
The relationship between the unique internal structure of biomimic woven fabric and its moisture management property is investigated using fractal derivative method. The biomimic fabric exhibits a fractal hierarchic inner structure, and its fractal hierarchy can be further extended by fleece finishing treatment on both surfaces of the fabric. Fractal derivative analysis indicates that the fuzzy biomimic fabric with a higher hierarchic construction after fleece finishing performs better in moisture permeability, and the result was proved by experimental tests.
\end{abstract}

\section{Introduction}

A woven fabric is generally produced by interlacing two sets of yarns, the warp and the weft which are at right angles to each other in the plane of cloth. The yarns constructing the fabric play an important part in transporting moisture from the microclimate between the skin and the garment to the outside atmosphere [1-3]. Therefore, a positive moisture transport property of cloth is critical in keeping the skin dry and promoting the comfort of clothing, particularly for the protective clothing used in extreme environments. For example, a moisture management ski suit should be able to effectively transfer the moisture released from human body during the motor process to the outer atmosphere to avoid cold feeling. In firefighting, rapid transport of the sensible and insensible perspiration through the clothing is crucial to extend the operation time of firefighters.

Tremendous methods have been promoted by researchers to improve the moisture permeation property of textile fabrics, such as choosing fibers with particular properties [46], arranging fibers in the yarn in several ways [7], and organizing in multiple ways the interlaced yarn within the fabric $[8,9]$.

Many elegant solutions to engineering problems have been inspired by biological phenomena [10, 11]. Much work in biologically inspired design involves specific cases of design that copy particular biological models. Fan et al. [12] developed a brand-new textile woven fabric with hierarchic inner structure imitating the configuration of plant. The weft yarns moved back and forth between the top layer and the bottom layer of the fabric providing a continuous path for water transpiration. In previous report, we promoted a new plant structured biomimic fabric by modifying the texture of Fan's fabric. Recently, we have chosen the warp yarns rather than the weft yarns to serve as the continuous path for water transpiration in a new biomimic fabric [13]. The number of warp yarns serving as the continuous path for moisture transportation was reduced from 24 to 12 , which facilitated manufacturing of the fabric by using a normal loom with 12 heald frames. In our experiments, the simplified fabric structure decreased half of the original moisture transport path. Experimental results indicated that the new fabric made a positive effect on the moisture permeability of the biomimic fabric. In this presentation, fractal derivative method was employed firstly to investigate the moisture management property of the biomimic fabric.

\section{Construction of the Biomimic Woven Fabric}

Our biomimic fabric includes three layers. Figure 1 demonstrates the interlacing law of the warp yarns in each layer of 


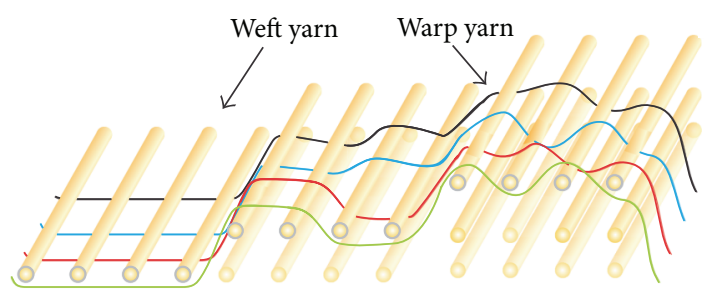

FIGURE 1: Schematic diagram of the original biomimic thermal fabric structure.

the fabric in a weaving repeat. The warp yarns in the bottom layer combine to form striped short floats in the fabric bottom surface. The same group of yarns split into pairs in the middle layer of the fabric forming $2 / 2$ matt weaves. In the top layer, the coupled yarns further separate into single yarn forming a $1 / 1$ plain weave. The warp yarns move back and forth between the top layer and the bottom layer in the fabric providing a continuous path for moisture transportation.

In order to simplify the fabric structure and give a mathematic description, the interlacing pattern of warp yarns was regarded as a tree-shaped model, shown in Figure 2(a). In our model, the striped short floats in the bottom layer of the fabric correspond to the trunk of tree, the pairs of warp yarns in the middle layer represent the two main branches, and the single warp yarn in the top layer stands for the slim branches.

After fleece finishing, the original biomimic fabric became a fuzzy fabric. Both fabric surfaces are covered with a fluff layer, which can be regarded as corresponding to the leaves and tiny roofs in the tree-shaped model. In this case, the hierarchic structure of the tree-shaped model is extended from three hierarchies to four hierarchies, shown in Figure 2(b).

\section{Fractal Characterization of the Biomimic Woven Fabric}

Fractal dimension of the biomimic woven fabric can be calculated. Suppose that the original scale equals the diameter of the trunk of tree, that is, the width of four yarns $4 d$. When the scale decreases to half size of the original scale, that is, the width of two yarns $2 d$, corresponding to the diameter of the main branch of the tree, the slim branch, leaves, and root can be ignored. Thus, the fractal dimension of the fabric is

$$
\alpha_{1}=\frac{\ln (1+2)}{\ln 2}=1.59
$$

When the scale decreases to a quarter of the original scale, that is, $d$, corresponding to the diameter of a single yarn, the leaves and roots layer of the fabric can be ignored. The fractal dimension of the fabric can be obtained:

$$
\alpha_{2}=\frac{\ln (3+8)}{\ln 4}=1.73
$$

For the fuzzy fabric after fleece finishing, when the scale decreases to one fortieth of the original scale, that is, $d / 10$, corresponding to the diameter of a single fiber, the fabric exhibits a four-level hierarchic structure. In the case that the yarn diameter is $40 \mathrm{~s} / 2$, that is, $301 \mu \mathrm{m}$ in diameter, the fiber diameter is $30 \mu \mathrm{m}$; there will be about 10 fibers on the surface of each warp interlacing point. After fleece finishing, a single fiber would be withdrawn from the yarn and broken into two fiber whiskers. Thus, the fractal dimension of the fabric is

$$
\alpha_{3}=\frac{\ln (11+8 \times 20+16 \times 20)}{\ln 40}=1.68 .
$$

The above analysis shows that the fractal dimension values in the main branching level, the slim branching level, and the tiny leaves and roofs level of the fabric are around 1.6. It is obvious that the scale affects the fractal construction of the fabric, which will inevitably impact the heat and moisture transfer process through the thickness direction of the fabric.

\section{Fractal Derivative for Moisture Permeability of Biomimic Woven Fabric}

The fractal derivative is a novel and effective analytical tool to deal with the heat and mass transfer problem in a discontinuous medium. Yang [14] proposed a definition expressed as

$$
f^{(\alpha)}\left(x_{0}\right)=\left.\frac{d^{\alpha} f(x)}{d x^{\alpha}}\right|_{x=x_{0}}=\lim _{x \rightarrow x_{0}} \frac{\Delta^{\alpha}\left(f(x)-f\left(x_{0}\right)\right)}{\left(x-x_{0}\right)^{\alpha}},
$$

where $\Delta^{\alpha}\left(f(x)-f\left(x_{0}\right)\right) \cong \Gamma(1+\alpha) \Delta\left(f(x)-f\left(x_{0}\right)\right)$.

$\mathrm{He}$ [15] introduced a new fractal derivative from the geometric point of view for engineering application defined as

$$
\frac{D u(t)}{D x^{\alpha}}=\frac{d u}{d s}=\lim _{\Delta x \rightarrow L_{0}} \frac{u(A)-u(B)}{k L_{0}^{\alpha}},
$$

where $k$ is a constant, $\alpha$ is the fractal dimension, $L_{0}$ is the smallest measure, and $d s$ is the distance between two points in the porous media.

Based on the fractal derivative theory, moisture permeation in the hierarchic fabric obeys fractal Fick's law expressed as

$$
q=\frac{K}{\mu} \frac{D P}{D x^{\alpha}}=\frac{K}{\mu} \lim _{\Delta x \rightarrow L_{0}} \frac{\Delta P}{k L_{0}^{\alpha}},
$$

where $q$ is the flux of moisture, $K$ the coefficient of permeability, $\mu$ is the coefficient of viscosity, $\Delta P$ is the pressure gradient, $\Delta x$ is a smaller measure scale, $k$ is a constant, $L_{0}$ is the smallest measure for moisture transfer path, and $\alpha$ is the fractal dimension of the moisture transfer path.

According to the above definition, moisture flux through the original three-layer biomimic fabric without fleece finishing can be expressed as

$$
q_{2}=\frac{K}{\mu} \frac{D P}{D x^{\alpha}}=\frac{K}{\mu} \lim _{\Delta x \rightarrow L_{2}} \frac{\Delta P}{k\left(L_{2}\right)^{\alpha_{2}}}=\frac{K}{\mu} \frac{\Delta P}{k\left(L_{2}\right)^{\alpha_{2}}} .
$$

Moisture flux through the three-layer fuzzy fabric after fleece finishing is

$$
q_{3}=\frac{K}{\mu} \frac{D P}{D x^{\alpha}}=\frac{K}{\mu} \lim _{\Delta x \rightarrow L_{3}} \frac{\Delta P}{k\left(L_{3}\right)^{\alpha_{3}}}=\frac{K}{\mu} \frac{\Delta P}{k\left(L_{3}\right)^{\alpha_{3}}},
$$




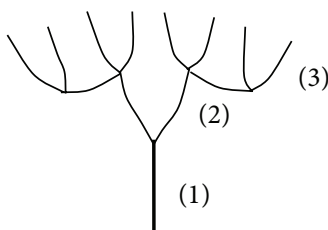

(a)

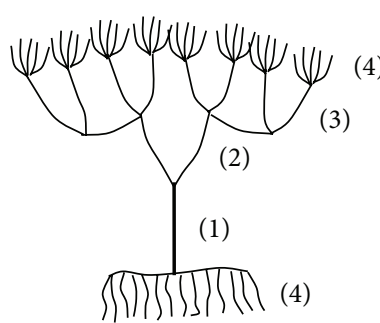

(b)

FIGURE 2: Tree-shaped model of the biomimic fabric, (a) original biomimic fabric with three hierarchies and (b) fuzzy biomimic fabric with four hierarchies: (1) trunk of the tree; (2) main branch of the tree; (3) slim branch of the tree; (4) leaves and roots of the tree.

where $L_{2}$ and $L_{3}$ are the smallest measure for moisture transfer path of the original biomimic fabric and the fuzzy fabric after fleece finishing, respectively. Suppose that the yarn cross-section is round; according to the geometric structure of the fabric, $L_{2}$ equals $2 \pi d$. $L_{3}$ equals the fluff height of the fuzzy fabric.

According to (7) and (8), the ratio between the moisture flux of the fuzzy biomimic fabric $q_{3}$ and the moisture flux of the original fabric $q_{2}$ is defined as moisture flux efficiency $\Phi$ and expressed as

$$
\Phi=\frac{q_{3}}{q_{2}}=\frac{(K / \mu)\left(\Delta P / k\left(L_{3}\right)^{\alpha_{3}}\right)}{(K / \mu)\left(\Delta P / k\left(L_{2}\right)^{\alpha_{2}}\right)}=\frac{\left(L_{2}\right)^{\alpha_{2}}}{\left(L_{3}\right)^{\alpha_{3}}} .
$$

Suppose the fluff height of the fleece finished fabric is $5 d$, about $1.5 \mathrm{~mm}$. Equation (9) became

$$
\begin{aligned}
\Phi & =\frac{q_{3}}{q_{2}}=\frac{(K / \mu)\left(\Delta P / k\left(L_{3}\right)^{\alpha_{3}}\right)}{(K / \mu)\left(\Delta P / k\left(L_{2}\right)^{\alpha_{2}}\right)}=\frac{\left(L_{2}\right)^{\alpha_{2}}}{\left(L_{3}\right)^{\alpha_{3}}}=\frac{(2 \pi d)^{1.73}}{(5 d)^{1.68}} \\
& =1.51 .
\end{aligned}
$$

Equation (10) indicates that the fuzzy biomimic fabric performs better than the original biomimic fabric in moisture transport efficiency. The result suggests that the moisture management property of biomimic fabric largely depends on the measure and the fractal hierarchy of the fabric. The functional performance of biomimic fabric can be regulated by setting a suitable hierarchic internal structure to the fabric.

\section{Moisture Permeability Tests of Biomimic Woven Fabric}

Two three-layer woolen biomimic fabrics were prepared for test: one was a fuzzy fabric with fluff height, $1.5 \mathrm{~mm}$ after fleece finishing, and the other was an original fabric without fleece finishing. Moisture resistant property of the two woolen fabrics was tested using a M925 Sweating Guarded Hotplate (SDL Atlas, USA). All the samples were conditioned for $48 \mathrm{~h}$ in the atmospheric conditions of temperature $20 \pm 2^{\circ} \mathrm{C}$ and relative humidity $65 \pm 2 \% \mathrm{RH}$, before the tests. Then specimens were put on the heated porous plate, which was covered by a water-vapor permeable but liquid-water impermeable membrane. Heat flux required to maintain a constant

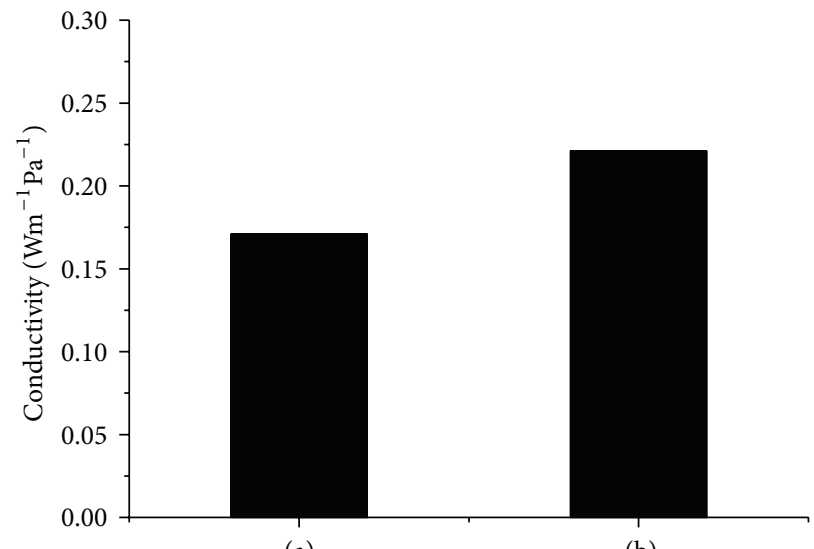

(a)

(b)

(a) Original biomimic fabric

(b) Fuzzy biomimic fabric

FIgURE 3: Moisture conductivity of biomimic woolen woven fabric.

temperature of the porous plate was recorded, and the moisture resistance of the fabrics was calculated according to the following equation:

$$
R_{\mathrm{et}}=\frac{A\left(p_{m}-p_{a}\right)}{H}-R_{\mathrm{e} t 0},
$$

where $R_{\mathrm{et}}$ is the moisture resistance of fabric, $A$ is the test area, $p_{m}-p_{a}$ is the moisture pressure difference between the measuring plate and the air, $H$ is the specific heat flux through the fabric, and $R_{\mathrm{et} 0}$ is the moisture resistance of the test apparatus.

In order to explain the intrinsic moisture transfer characteristic of the fabric samples, moisture conductivity was calculated using the following equation:

$$
q=\frac{h}{R_{\mathrm{et}}},
$$

where $q$ is the moisture conductivity of fabric samples and $h$ is the thickness of fabric sample.

The calculated moisture conductivity of the two fabrics shown in Figure 3 suggests that fleece finish does have a positive effect on the moisture management of the biomimic fabric. Moisture conductivity of the fuzzy biomimic fabric is 
about 1.29 times of that of the original fabric. The deviation between the test result and the fractal derivative theoretical analysis is about $14 \%$, indicating that fractal derivative approach is a promising method to be used in optimizing the internal structure of the hierarchic woven fabric.

\section{Conclusions}

Moisture permeability of biomimic woven fabric with fractal characteristic was investigated using fractal derivative method. Fractal derivative analysis indicated that the fuzzy fabric after fleece finishing performs better in moisture management comparing with the original fabric without fleece finishing, and the result was proved by experimental test. The functional performance of biomimic fabric significantly depends on its hierarchic internal structure. Fractal derivative provides a novel method for optimalizing the structure of hierarchic textile materials.

\section{Conflict of Interests}

The authors declare that there is no conflict of interests regarding the publication of this paper.

\section{Acknowledgments}

The work is supported by National Natural Science Foundation of China under Grants nos. 51203114 and 11372205, Special Program of China Postdoctoral Science Foundation Grant no. 2013T60559, China Postdoctoral Science Foundation under Grants no. 2012M521122, Postdoctoral Science Foundation Jiangsu Province Grant no. 1301151C, Natural Science Funds of Tianjin under Grant no. 12JCQNJC01500, Program for New Century Excellent Talents in University under Grants no. NCET-12-1063, College Students' Innovative Entrepreneurial Training Plan Program of Tianjin Polytechnic University (Zhi Liu), and PAPD (a project funded by the Priority Academic Program Development of Jiangsu Higher Education Institutions).

\section{References}

[1] K. Min, Y. Son, C. Kim, Y. Lee, and K. Hong, "Heat and moisture transfer from skin to environment through fabrics: a mathematical model," International Journal of Heat and Mass Transfer, vol. 50, no. 25-26, pp. 5292-5304, 2007.

[2] X. Qian and J. Fan, "A quasi-physical model for predicting the thermal insulation and moisture vapour resistance of clothing," Applied Ergonomics, vol. 40, no. 4, pp. 577-590, 2009.

[3] J. Fan and J.-H. He, "Biomimic design of multi-scale fabric with efficient heat transfer property," Thermal Science, vol. 16, no. 5, pp. 1349-1352, 2012.

[4] R. Bagherzadeh, M. Gorji, M. Latifi, P. Payvandy, and L. X. Kong, "Evolution of moisture management behavior of highwicking 3D warp knitted spacer fabrics," Fibers and Polymers, vol. 13, no. 4, pp. 529-534, 2012.

[5] J. Fan and X. Shang, "Water permeation in the branching channel net of wool fiber," Heat Transfer Research, vol. 44, no. 5, pp. 465-472, 2013.
[6] J.-H. He, Q.-L. Wang, and J. Sun, “Can polar bear hairs absorb environmental energy?” Thermal Science, vol. 15, no. 3, pp. 911913, 2011.

[7] N. Özdil, A. Marmarali, and S. D. Kretzschmar, "Effect of yarn properties on thermal comfort of knitted fabrics," International Journal of Thermal Sciences, vol. 46, no. 12, pp. 1318-1322, 2007.

[8] G. Bedek, F. Salaün, Z. Martinkovska, E. Devaux, and D. Dupont, "Evaluation of thermal and moisture management properties on knitted fabrics and comparison with a physiological model in warm conditions," Applied Ergonomics, vol. 42, no. 6, pp. 792-800, 2011.

[9] A. Majumdar, S. Mukhopadhyay, and R. Yadav, "Thermal properties of knitted fabrics made from cotton and regenerated bamboo cellulosic fibres," International Journal of Thermal Sciences, vol. 49, no. 10, pp. 2042-2048, 2010.

[10] A. V. Singh, A. Rahman, N. V. G. S. Kumar et al., "Bio-inspired approaches to design smart fabrics," Materials and Design, vol. 36, pp. 829-839, 2012.

[11] L. H. Shu, K. Ueda, I. Chiu, and H. Cheong, "Biologically inspired design," CIRP Annals-Manufacturing Technology, vol. 60, no. 2, pp. 673-693, 2011.

[12] J. T. Fan, M. K. Sarkar, Y. C. Szeto, and X. M. Tao, "Plant structured textile fabrics," Materials Letters, vol. 61, no. 2, pp. 561-565, 2007.

[13] J. Fan, Q. Cheng, L.-Y. Zhao, Y. Liu, and C.-Q. Ma, "A biomimic thermal fabric with high moisture permeability," Thermal Science, vol. 17, no. 5, pp. 1425-1430, 2013.

[14] X.-J. Yang, H. M. Srivastava, J.-H. He, and D. Baleanu, "Cantortype cylindrical-coordinate method for differential equations with local fractional derivatives," Physics Letters A, vol. 377, no. 28-30, pp. 1696-1700, 2013.

[15] J.-H. He, "A new fractal derivation," Thermal Science, vol. 15, supplement 1, pp. S145-S147, 2011. 


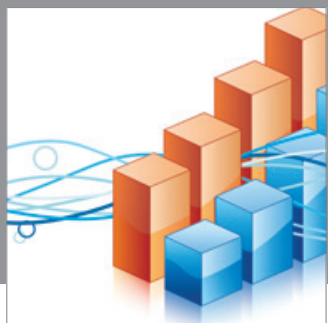

Advances in

Operations Research

mansans

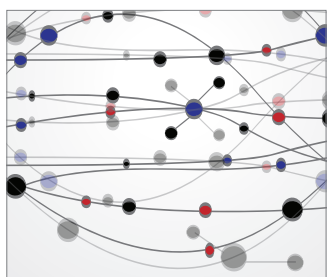

The Scientific World Journal
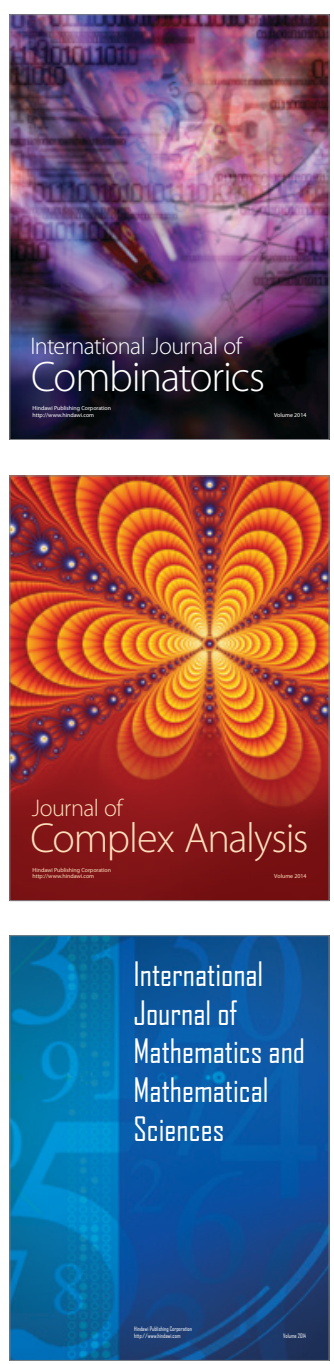
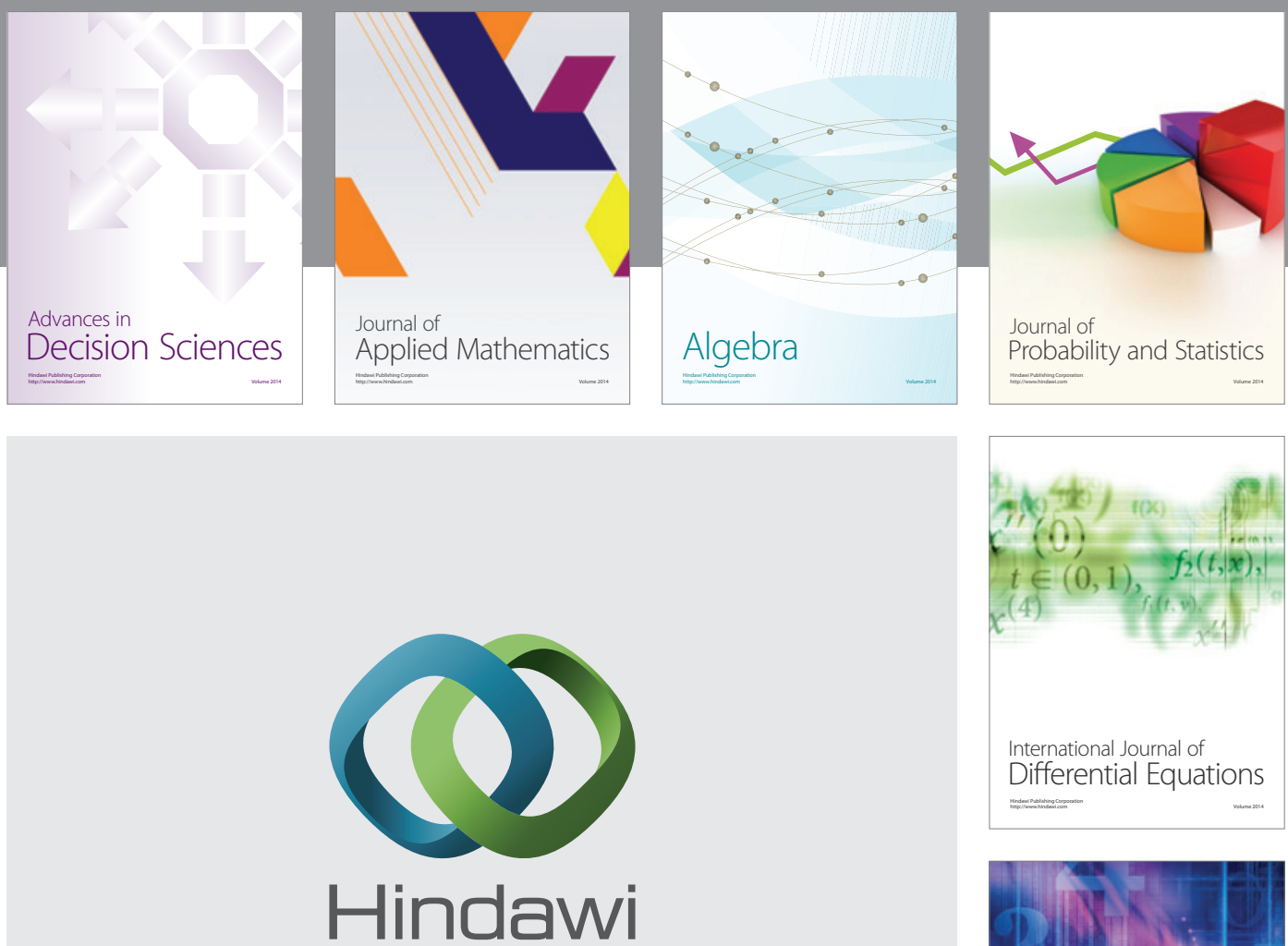

Submit your manuscripts at http://www.hindawi.com
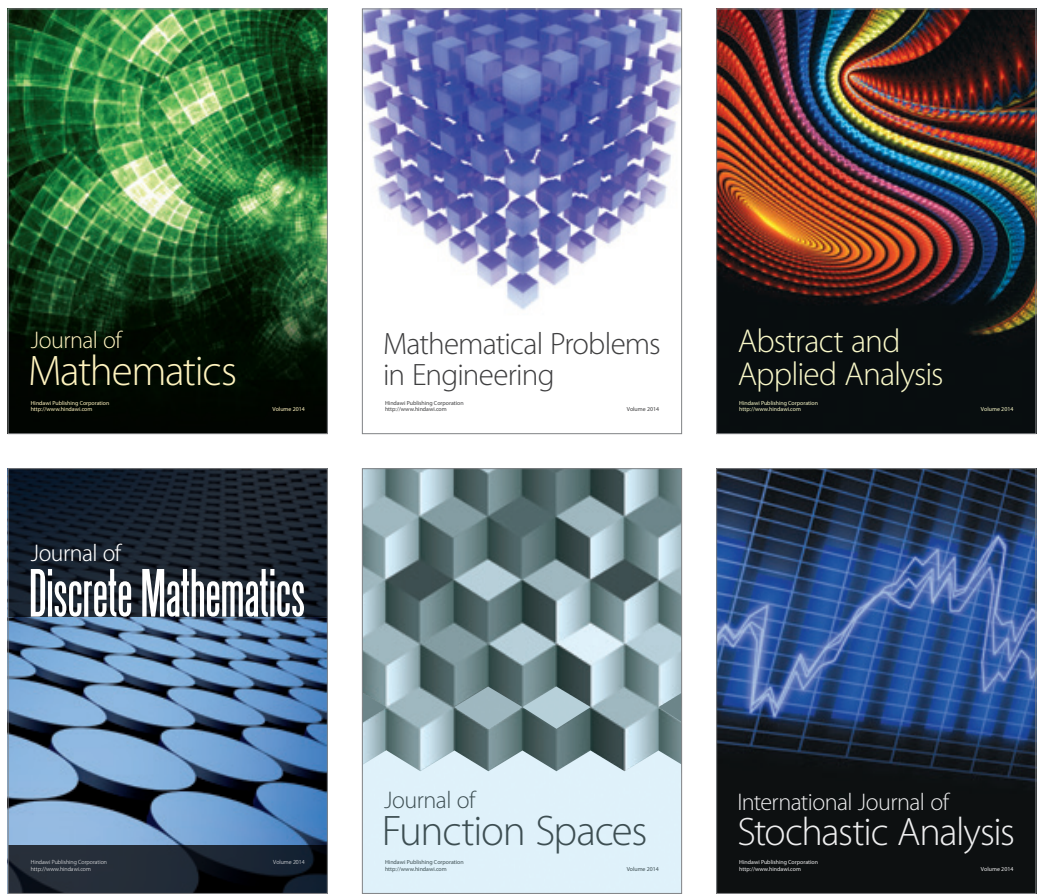

Journal of

Function Spaces

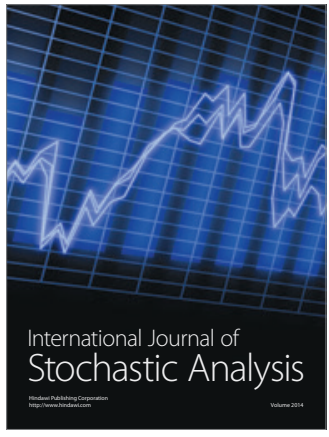

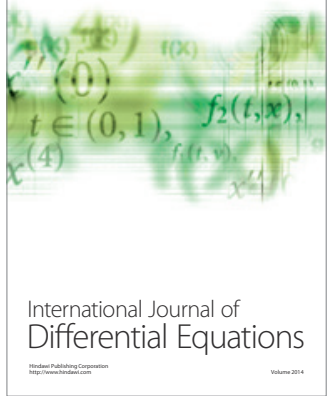
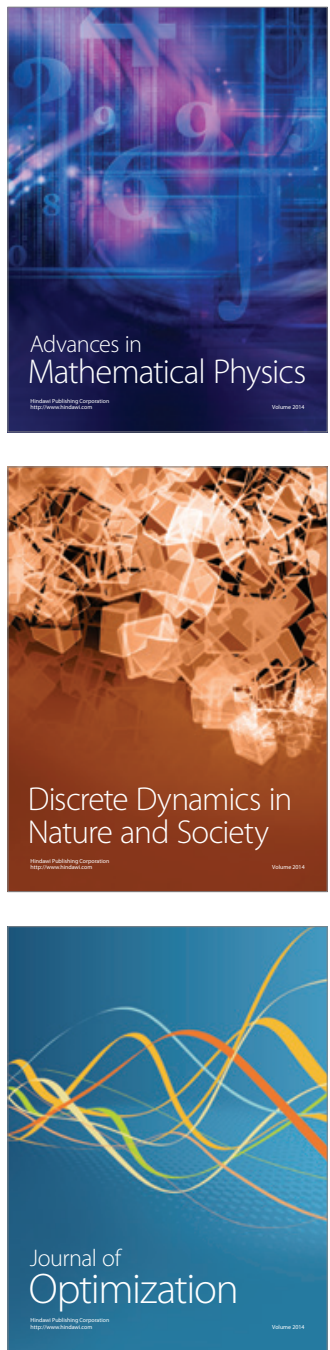